\title{
Reported Incidences of Postoperative Delirium in State and National Hospital Claims Databases
}

\author{
Khaled Al-Robaidia ${ }^{\circledR}$, Muruga Loganathan ${ }^{\mathrm{b}}$, Laith Alhuneafat ${ }^{\mathrm{a}}$, Samir S. Al-Ghazawi ${ }^{\mathrm{a}}$, \\ Keith Vogt ${ }^{\mathrm{c}}$, Parthasarathy D. Thirumala ${ }^{\mathrm{a}, \mathrm{d}, \mathrm{e}}$
}

\begin{abstract}
Background: The aim of the study was to evaluate the incidence of postoperative delirium (POD) following a comprehensive list of surgical procedures utilizing data from state and national inpatient hospital databases.
\end{abstract}

Methods: The incidence of POD occurring during surgical hospital admissions was evaluated across 15 surgical categories, and different patient demographics using California State Inpatient Database (SID) and National Inpatient Sample (NIS).

Results: Analysis of 4 years of SID sample resulted in 47,875 cases of POD with an overall incidence of $1.06 \%$. The incidence was highest following transplant surgeries $6.82 \%$, musculoskeletal surgeries $1.95 \%$, neurological surgeries $1.81 \%$ and cardiac surgeries $1.81 \%$. The mean age of patients with delirium was $71.6 \pm 15.3$ (standard deviation (SD)) compared with $47.8 \pm 23.6(\mathrm{SD})$ in patients without delirium. Analysis of the NIS database resulted in an annual incidence of $1.78 \%$, estimating at least 200,000 cases of POD occurring every year in the USA.

Conclusions: This study highlights the size and variation of postoperative incidence across different patient characteristics, risk factors, surgical procedures, and research methods. Delirium occurring in the postsurgical period remains to be a serious potentially preventable problem affecting hospitals worldwide. Implementing additional measures for early detection and prevention can help lessen the morbidity and mortality burden associated with delirium.

Keywords: Delirium; Postoperative; Incidence; National Inpatient

Manuscript submitted May 6, 2021, accepted October 27, 2021

Published online December 15, 2021

aDepartment of Neurology, University of Pittsburgh, Pittsburgh, PA, USA ${ }^{b}$ Department of Child Adolescent Psychiatry, Chestnut Ridge Counseling Services, Uniontown, PA, USA

'Department of Anesthesiology, University of Pittsburgh, Pittsburgh, PA, USA dDepartment of Neurological Surgery, University of Pittsburgh, Pittsburgh, PA, USA

${ }^{\text {eC }}$ Corresponding Author: Parthasarathy D. Thirumala, Center for Clinical Neurophysiology, Department of Neurological Surgery, UPMC Presbyterian-Suite B-400, 200 Lothrop Street, Pittsburgh, PA 15213, USA.

Email: thirumalapd@upmc.edu

doi: https://doi.org/10.14740/jnr675
Sample; State Inpatient Sample; Underdiagnosis; Underreported; Surgery

\section{Introduction}

Postoperative delirium is an acute phase of confusion within $72 \mathrm{~h}$ after surgery [1-3]. Despite being a common and frequently unrecognized condition, postoperative delirium displays wide variation in reported incidences [4]. In 2010, over 50 million inpatient surgical procedures were performed in the USA alone and that number is increasing annually [5]. In a systematic review of elderly patients who underwent surgical procedures, the incidence of delirium ranged between $11 \%$ and $46 \%$ in cardiac surgeries, $13-50 \%$ in noncardiac surgeries, and $12-51 \%$ in orthopedic surgeries [6]. Currently, the reports of incidence in the literature vary too widely; and as delirium is multifactorial, controlling every factor seems difficult [7]. This variation in incidence could potentially mean that numerous patients with delirium go unrecognized leading to significant consequences.

In the short term, delirium after surgery increases the length of hospital stay, the number of days of mechanical ventilation, and the length of intensive care unit (ICU) stay [8, 9]. Furthermore, in-hospital delirium is associated with two and a half times increase in 1-year medical costs [10]. As a longterm consequence, it increases mortality, increases the risk of further hospitalizations, worsens cognitive function, and increases the risk of dementia by 10 folds [3, 11-14]. Delirium in the intensive care setting is of a particular importance, as patients in the ICU tend to be more acutely ill, older, and have a higher risk to be mechanically ventilated which should reflect in a higher incidence of delirium [15].

The goal of this project was to comprehensively evaluate the incidence of delirium following a wide range of surgical procedures. To achieve this goal, the incidence of delirium was evaluated throughout surgical admissions using statewide and nationwide in-hospital databases from the USA. The use of inpatient claims databases in research can provide incidences across a large patient population of different characteristics, and in multiple clinical settings. Another aim was to compare the resulted incidences with incidences reported in medical literature to provide an idea on the fidelity of hospital claims databases in documenting postoperative outcomes. 


\section{Materials and Methods}

\section{Data source}

California State Inpatient Database (SID) and National Inpatient sample (NIS) obtained from healthcare cost utilization project (HCUP) were individually analyzed in this study to evaluate the incidence of delirium following different surgical procedures. The SID encompasses about $97 \%$ of California state community hospital inpatient discharge records. It has more than 100 clinical and nonclinical variables including patient demographics, diagnoses (primary, secondary), list of procedures performed, admission status, discharge status, length of stay and comorbidities. SID is also unique in having present on admission (POA) variable which is valuable in distinguishing whether a patients' diagnosis was recorded prior to admission or resulted as a complication during hospitalization. The NIS is the largest publicly available all-payer inpatient healthcare database in the USA, yielding national estimates of hospital inpatient stays. Unweighted, it contains data from more than 7 million hospital stays each year. Weighted, it estimates more than 35 million hospitalizations nationally, allowing for the study of common and uncommon diagnoses and procedures on a national level [16]. The NIS lacks the POA variable which makes it less useful when utilized for evaluating hospital complications when compared to the SID. Data were acquired for the years 2008 - 2011 as they were the only years available for accusation for the California SID by the time the study was performed. International Classification of Diseases, Ninth Revision Clinical Modification (ICD-9-CM) codes and Clinical Classification Software (CCS) codes were used for defining our included diagnoses and procedures. This study was reviewed by the Institutional Review Board (IRB) of the University of Pittsburgh and determined to be exempt as there was no direct contact with humans neither there were any patient identifiers in the data. Ethical compliance with human study was exempted by the IRB.

\section{Outcomes studied}

The primary outcome for this study was the incidence of delirium occurring in the postoperative period. This period was defined in this project as the period during hospitalization for any elective surgical intervention and before discharge from the hospital. Postoperative delirium was defined by the presence of at least one of the following ICD-9-CM diagnoses codes: (293.9, 780.09, 298.9, 290.11, 290.3, 290.41, 290.20, 293, 293.0, 293.1, 293.8, 293.81, 293.82, 292.81, 291.0, 292.11, 292.12, 348.3, 348.31, 348.39, 349.82, 437.2), plus a negative POA variable value. A similar definition was used for the NIS database but without a negative POA (postoperative delirium) variable given that this variable was not available for the NIS. The broad selection of codes for delirium was due to our awareness of underrecognition and inaccuracies in coding, and to detect the majority of delirium cases occurring following surgery.

\section{Covariates}

The surgical datasets included a wide variety of patient demographics representing the state of California in the SID and the whole USA in the NIS including different age groups from 0 to 100 , gender, and race. Clinical covariates encompassed 29 comorbidities based on the Elixhauser comorbidity index including congestive heart failure, valvular disease, pulmonary circulation and chronic pulmonary disorders, peripheral vascular disease, hypertension, paralysis and other neurological disorders, diabetes, hypothyroidism, renal failure, liver disease, tumors, weight changes, anemias, fluid and electrolyte disorders, psychosis, alcohol abuse, depression, and rheumatological diseases. No ICD-9 codes were used to define these comorbidities as they were already coded in the datasets as binary variables from their origin.

\section{Statistical analysis}

Dataset construction was performed using SAS 9.4. Individual analyses were performed for each dataset (SID/NIS). The main datasets for the years 2008 - 2011 were limited to elective surgical admission records and diagnostic procedures utilizing dataset variables that distinguish the type of admission. Surgical data for each year were then merged into a common dataset that was used for analysis. Every surgical record in the datasets contained up to 25 reported diagnoses with a corresponding POA variable. The first diagnosis variable in each record was considered as the primary admitting diagnosis and the other 24 diagnoses were considered as secondary diagnoses. The surgical records were divided into 15 surgical categories according to the organ system involved to make the data presentation more meaningful. A SAS macro was written and utilized to search through all the included surgical records for the diagnosis of postoperative delirium and calculate incidences across the defined patient characteristics. All reported incidences were based on the division of the numbers of delirium cases over the number of surgical procedures performed. The results were reported as "number of procedures" not as a number of patients as the same patient may have undergone several procedures during the included time frame. It was not possible to limit the datasets to unique patients as this was deidentified data that did not contain patient identifiers. A year-by-year analysis was also performed to observe the trends from 2008 to 2011. Data analysis in the project was largely descriptive with no variable adjustment due to several limitations; however, a limited independent sample $t$-test was performed to produce statistical comparison between numerical variables including age and length of stay, and Chi-square test was used to compare between categorical variables including gender. The same process was replicated for the NIS database. In addition, the NIS was utilized to produce national incidence estimates by weighing the included records to replicate the actual population of the USA. Weigh- 


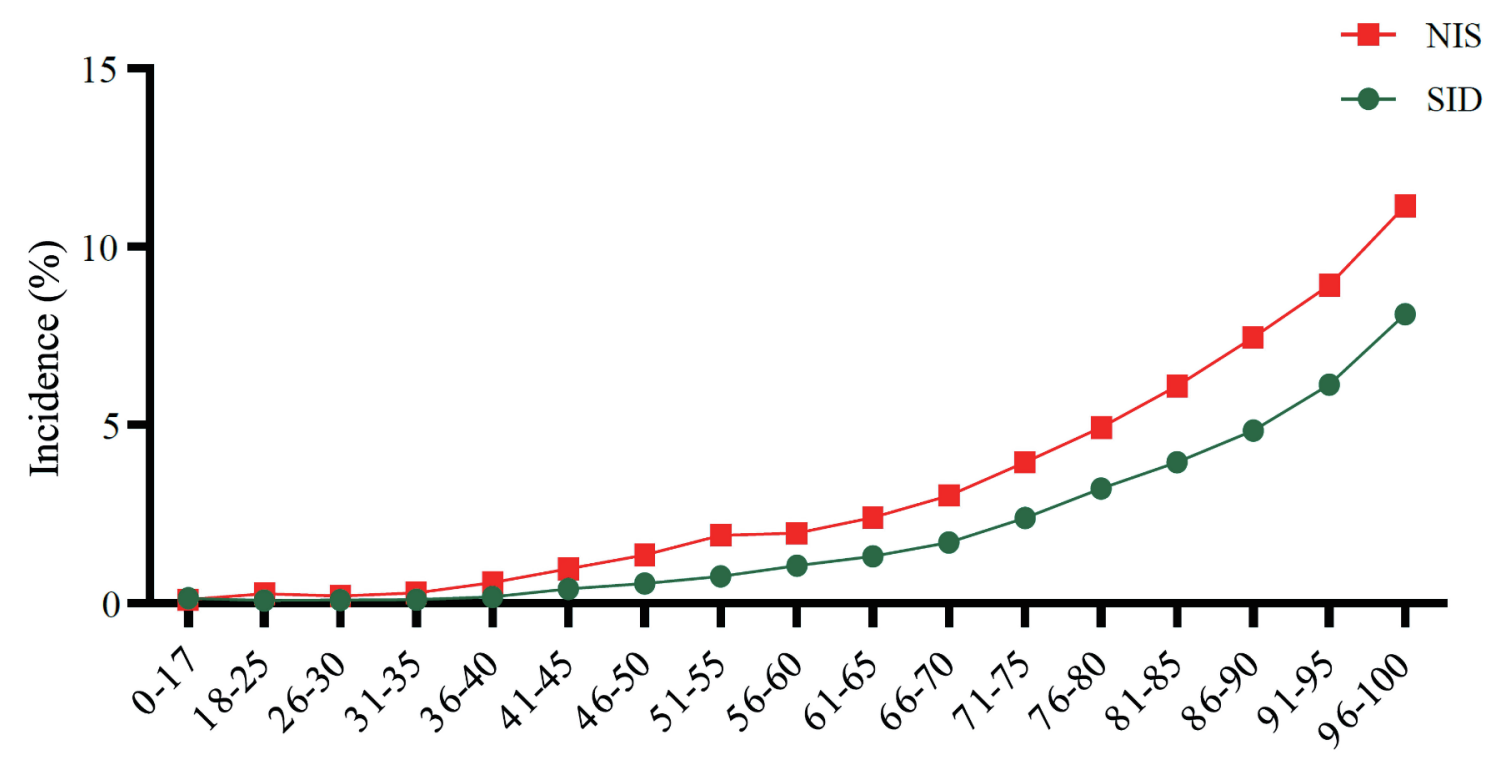

Figure 1. Postoperative delirium incidence across 17 different age groups. SID: State Inpatient Database; NIS: National Inpatient Sample.

ing was performed using weighing macros available on the HCUP website.

\section{Results}

\section{SID}

\section{Patient characteristics}

A total of 4,507,860 inpatient discharge records (mean age: $48.1 \pm 23.7$ standard deviation (SD)) spanning the years 2008 through 2011 were identified from the SID. Each year of the SID contained an average of 1,126,965 surgical discharge records. Our study population included $39.2 \%$ males and $60.8 \%$ females. White race was the most prevalent race $(54.1 \%)$ followed by Hispanic race 29\%, Asian 7.9\%, African American $6.6 \%$, and others $2.4 \%$. Of our sample, $43.5 \%$ had more than one comorbidity prior to surgery. Most common performed surgical procedures were obstetrics and gynecology (OBGYN) surgeries $(26.64 \%)$, followed by musculoskeletal $21.47 \%$, and gastrointestinal surgeries $16.78 \%$. Of the included surgical records, $0.6 \%$ were missing age value, $2.1 \%$ were missing gender, and $6.7 \%$ were missing race.

\section{Incidence of delirium}

The overall rate of postoperative delirium found in the SID was $1.06 \%(n=47,875)$. On average 11,967 cases of postoperative delirium occurred every year in California state, and incidences did not vary significantly across the years. Incidence of delirium was highest following transplant surgeries at $6.82 \%$, musculoskeletal surgeries $1.95 \%$, neurological surgeries $1.81 \%$ and cardiac surgeries $1.81 \%$. OBGYN surgeries including caesarean section had the lowest incidence of postoperative delirium at $0.06 \%$. Mean age of patients with postoperative delirium was $71.6 \pm 15.3(\mathrm{SD})$ compared with $47.8 \pm 23.6(\mathrm{SD})$ in patients who did not develop delirium ( $\mathrm{P}$ value $<0.001)$. For patients who developed delirium, $51.1 \%$ were males compared to $48.9 \%$ in females, and $72.8 \%$ were White followed by $14.1 \%$ Hispanics, 5.8\% Asians and 5.4\% African Americans. Mean length of stay for patients with delirium was 14.3 days compared to 4.6 days in patients without delirium ( $\mathrm{P}$ value $<0.001)$.

The incidence of postoperative delirium increased progressively with age exhibiting a rate almost two times higher every 15 years increase of age especially in ages above 60 . In year 2011, surgical patients who were 56 - 60 years of age developed delirium at a rate of $1.06 \%$, while those who were 71 - 75 of age developed delirium at a rate of $2.4 \%$. Finally, patients who were 80 years or older developed delirium at a rate of more than $4 \%$ (Fig. 1). Incidence of postoperative delirium was higher in males $(1.42 \%)$ compared to $0.88 \%$ in females ( $\mathrm{P}$ value $<0.001$, Table 1$)$. Incidences were comparable between males and females in several types of surgeries, but we found increased incidences in males following neurosurgical, gastrointestinal, blood, and urological surgeries while females had higher incidences following musculoskeletal surgeries. Moreover, incidence of postoperative delirium in Whites was $1.74 \%$ compared to $0.87 \%$ in African Americans and $0.41 \%$ in Hispanics in year 2011 (Table 1).

As the included surgical categories were broad in nature, a closer assessment was done to evaluate incidences after specific surgical procedures (Table 2). Of all included surgeries, transplant surgeries were associated with the highest rates of postoperative delirium. Among the transplant surgeries, combined heart-lung transplant surgeries were associated with the highest incidence of postoperative delirium (17.95\%), followed by surgeries on the abdominal aorta at $10.28 \%$ and uni- 
Table 1. Comparison of the Demographics and the Incidence of Delirium in the Years of 2008 and 2011 (SID Database)

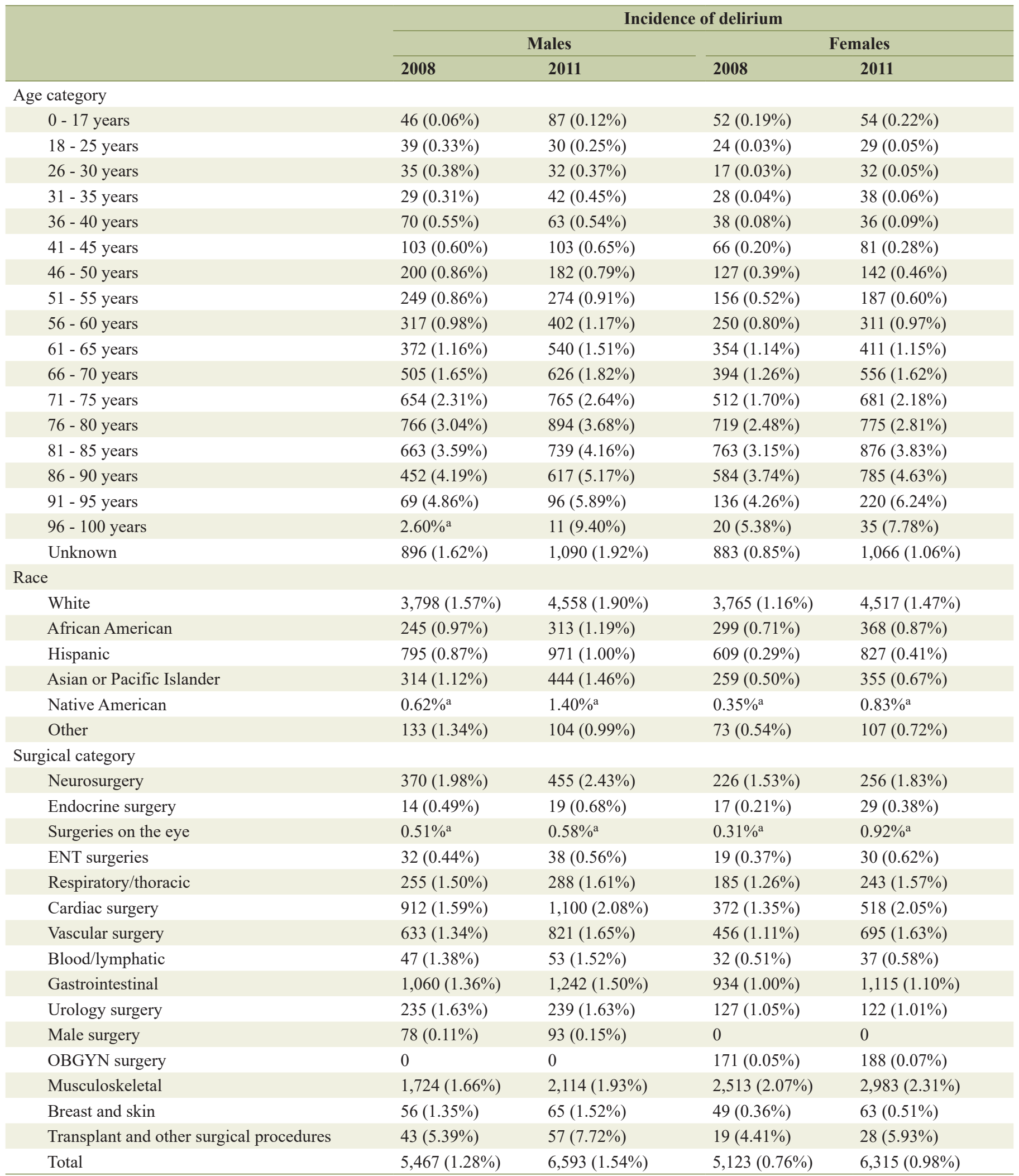

aNumber of patients $(<10)$ is too small to be reported. Data presentation: number of delirium cases (delirium incidence (\%) after all surgical procedures). SID: State Inpatient Database; OBGYN: obstetrics and gynecology. 
Table 2. Selected Surgeries Associated With a High Incidence of Delirium (SID Database)

\begin{tabular}{|c|c|c|c|}
\hline \multirow{2}{*}{ Type of surgery } & \multicolumn{3}{|c|}{ Incidence of postoperative delirium } \\
\hline & Males & Females & Total \\
\hline \multicolumn{4}{|l|}{ Neurosurgery } \\
\hline Excision or destruction of lesion or tissue of brain & $299(3.53 \%)$ & $207(2.67 \%)$ & $506(2.99 \%)$ \\
\hline Excision of lesion or tissue of cerebral meninges & $72(4.73 \%)$ & $104(3.18 \%)$ & $176(3.52 \%)$ \\
\hline \multicolumn{4}{|l|}{ Cardiac surgery } \\
\hline Open and other replacement of aortic valve with tissue graft & $689(6.47 \%)$ & $360(5.84 \%)$ & $1,049(6.23 \%)$ \\
\hline (Aorto) coronary bypass of three coronary arteries & $453(4.05 \%)$ & $130(3.82 \%)$ & $583(3.99 \%)$ \\
\hline (Aorto) coronary bypass of two coronary arteries & $454(3.89 \%)$ & $163(3.76 \%)$ & $617(3.85 \%)$ \\
\hline (Aorto) coronary bypass of four or more coronary arteries & $266(4.32 \%)$ & $58(4.02 \%)$ & $324(4.26 \%)$ \\
\hline Implant of pulsation balloon & $193(3.41 \%)$ & $62(2.50 \%)$ & $255(3.13 \%)$ \\
\hline Single internal mammary-coronary artery bypass & $161(3.69 \%)$ & $48(3.09 \%)$ & $209(3.53 \%)$ \\
\hline Insertion of implantable heart assist system & $49(10.43 \%)$ & $9(7.56 \%)$ & $58(9.85 \%)$ \\
\hline \multicolumn{4}{|l|}{ Vascular surgery } \\
\hline Resection of vessel with replacement, aorta, abdominal & $209(10.50 \%)$ & $71(9.78 \%)$ & $280(10.28 \%)$ \\
\hline Resection of vessel with replacement, thoracic vessels & $84(7.40 \%)$ & $41(6.31 \%)$ & $125(6.98 \%)$ \\
\hline Interruption of the vena cava & $331(2.64 \%)$ & $269(2.05 \%)$ & $600(2.34 \%)$ \\
\hline Clipping of aneurysm & $71(7.24 \%)$ & $122(5.21 \%)$ & $193(5.73 \%)$ \\
\hline Internal fixation of bone without fracture reduction, femur & $135(3.44 \%)$ & $275(4.00 \%)$ & $410(3.61 \%)$ \\
\hline Dorsal and dorsolumbar fusion of the posterior column, posterior technique & $156(3.89 \%)$ & $199(3.87 \%)$ & $355(3.81 \%)$ \\
\hline Other amputation below knee & $295(4.18 \%)$ & $171(5.08 \%)$ & $467(4.46 \%)$ \\
\hline Amputation above knee & $165(5.14 \%)$ & $121(4.47 \%)$ & $286(4.82 \%)$ \\
\hline Total knee replacement & $1,192(1.66 \%)$ & $1,696(1.45 \%)$ & $2,900(1.49 \%)$ \\
\hline \multicolumn{4}{|l|}{ Urology surgery } \\
\hline Radical cystectomy & $179(6.28 \%)$ & $32(6.05 \%)$ & $211(6.23 \%)$ \\
\hline Percutaneous nephrostomy without fragmentation & $107(2.06 \%)$ & $107(1.73 \%)$ & $214(1.87 \%)$ \\
\hline Other transurethral excision or destruction of lesion or tissue of bladder & $112(1.39 \%)$ & $38(1.43 \%)$ & $150(1.38 \%)$ \\
\hline Partial nephrectomy & $39(1.27 \%)$ & $33(1.49 \%)$ & $72(1.31 \%)$ \\
\hline \multicolumn{4}{|l|}{ Gastrointestinal } \\
\hline Other partial resection of small intestine & $404(3.82 \%)$ & $472(3.86 \%)$ & $877(3.8 \%)$ \\
\hline Open and other right hemicolectomy & $405(4.16 \%)$ & $385(3.39 \%)$ & $790(3.7 \%)$ \\
\hline Open and other sigmoidectomy & $324(3.41 \%)$ & $300(3.05 \%)$ & $624(3.18 \%)$ \\
\hline Other lysis of peritoneal adhesions & $249(3.09 \%)$ & $289(2.31 \%)$ & $540(2.59 \%)$ \\
\hline
\end{tabular}


Table 2. Selected Surgeries Associated With a High Incidence of Delirium (SID Database) - (continued)

\begin{tabular}{|c|c|c|c|}
\hline \multirow{2}{*}{ Type of surgery } & \multicolumn{3}{|c|}{ Incidence of postoperative delirium } \\
\hline & Males & Females & Total \\
\hline Cholecystectomy & $224(2.40 \%)$ & $142(1.43 \%)$ & $366(1.87 \%)$ \\
\hline Radical pancreaticoduodenectomy & $112(6.01 \%)$ & $84(5.14 \%)$ & $196(5.56 \%)$ \\
\hline Partial gastrectomy with anastomosis to jejunum & $92(5.51 \%)$ & $50(3.16 \%)$ & $142(4.33 \%)$ \\
\hline Bilateral lung transplantation & $30(11.28 \%)$ & $16(7.84 \%)$ & $46(9.79 \%)$ \\
\hline Combined heart-lung transplantation & $17.65 \% \mathrm{a}$ & $18.18 \% \mathrm{a}$ & $17.95 \% \mathrm{a}$ \\
\hline Heart transplantation & $35(4.69 \%)$ & $11(3.93 \%)$ & $46(4.47 \%)$ \\
\hline Other transplant of liver & $124(7.22 \%)$ & $67(6.71 \%)$ & $191(7.02 \%)$ \\
\hline
\end{tabular}

aNumber of patients $(<10)$ is too small to be reported. Data presentation: number of delirium cases (delirium incidence (\%) after individual surgical procedure). SID: State Inpatient Database.

lateral lung transplant $10.13 \%$.

Lastly, most frequent presurgical morbidities in patients who developed delirium were: hypertension $35 \%$, diabetes $16 \%$, deficiency anemia $15 \%$, chronic lung disease $11 \%$, and obesity $10 \%$. A progressive rise in delirium incidences was noted with the rise in number of presurgical comorbidities the patient had.

\section{NIS}

Analysis of 46,747,030 weighted discharge records for nationwide admissions between 2008 and 2011 yielded an overall rate of postoperative delirium of $1.78 \%(n=829,837)$. Females compromised $58 \%$ of the sample. Whites were the most prevalent race $(69.2 \%)$, followed by African Americans $12.1 \%$, Hispanics $11.6 \%$ and all other races $7.1 \%$. Incidence of delirium was highest following transplant surgeries at $8.19 \%$, vascular surgeries $3.77 \%$, respiratory/thoracic surgeries $3.40 \%$, and surgeries on the eye $3.34 \%$. Incidence of delirium following cardiac surgeries was $2.21 \%$.

On average, more than 200,000 nationwide cases a year of postoperative delirium were found on analysis of the weighted NIS data. Number of cases increased from 180,281 cases in year 2008 to 233,841 in year 2011. Similar to the observed trend in the SID, a progressive rise in the incidence of postoperative delirium was noted as patients' age and number of comorbidities increased. In year 2011, those who were 56 - 60 of age developed postoperative delirium at a rate of $1.97 \%$ compared to incidence rate of more than $6 \%$ in ages above 80 (Fig.1). Postoperative delirium was found in $2.0 \%$ of Whites compared to $1.6 \%$ in African Americans, and $1.0 \%$ in Hispanics.

\section{Incidences in coronary artery bypass and hip surgeries}

A subanalysis and comparison between several high-risk and common procedures was performed on the SID and NIS. Upon analysis of surgeries performed in California in 2011, there were 12,261 coronary artery graft bypass (CABG) surgeries and 41,091 hip surgeries performed with $4.24 \%$ and $2.93 \%$ incidence of delirium respectively. Compared to the SID, analysis of the NIS for the same year resulted in 163,764 CABG and 464,502 hip surgeries performed nationwide with a $4.83 \%$ and $3.83 \%$ incidence of delirium respectively. Based on the previous results, it can be expected that in any given year, there would be at least an approximately 8,000 cases of delirium following CABG and 17,800 cases of delirium following hip surgery on a national basis.

\section{Discussion}

Delirium is one of the commonly missed postoperative complications that can be associated with significant morbidity and mortality. To our knowledge, this is the first report to review the incidence of postoperative delirium across a large scale of different surgical procedures and patient demographics. Our analysis of the largest public inpatient datasets available in the USA (SID/NIS), we found a postoperative delirium incidence rate of $1.06 \%$ with an average of 11,967 cases occurring every year in the state of California. Analysis of the NIS resulted in a $1.78 \%$ incidence of postoperative delirium with an estimate of 200,000 cases occurring nationally in the USA every year.

Incidences reported in this review were lower when compared to incidences reported in medical literature [17-19]. This could be attributed to several reasons. First, throughout this project our aim was to include the vast majority of billable surgical procedures whether invasive or minimally invasive procedures. This criterion resulted in the inclusion of a large number of lowrisk routine procedures, for example, cesarean sections. On the other hand, published papers in medical literature mainly tended to focus on the incidence of delirium in only high-risk surgical procedures, patients in the ICU setting, or in older patients with many presurgical comorbidities, which realistically should result in higher delirium incidences. Interestingly, we found that larger scale published papers that included a higher number of 
patients of different ages and presurgical risk factors tended to have incidences comparable to our reported numbers [20-24]. Second, even when we took into consideration the number of comorbidities each patient has and evaluated a subset of highrisk operations, incidences were still lower when compared with reported incidences for similar procedures. This could be due to hospital claims databases being primarily used for billing purposes which is prone to errors in coding and under-reporting [25]. Whereas, in prospective studies care providers are actively tracking and implementing diagnostic criteria to detect any delirium event thus giving a more accurate estimate of a higher incidence of delirium. McCoy et al reported in their review that delirium in hospital claims databases may be underreported by 10 -fold or more when comparing it with published rates of delirium, attributing this variation to the possibility of failure in coding or diagnosis [26]. It is worthy to mention that the observed trend of increasing number of delirium cases every year from 2008 to 2011 in the SID and NIS may be attributed to the increased quality and accuracy of data reporting and not necessarily mean that delirium is on the rise.

This study signifies the importance of the POA variable in claims data. Incidences were almost two times higher in the NIS compared to the SID. This variation could be explained by differences in the demographics and diagnoses-reporting metrics in both databases, but also could be attributed to the deficiency of POA variable in the NIS which resulted in including cases of a previous history of delirium plus delirium occurring as a complication after surgery, giving rise to a possible measurement bias in the results of the NIS. We tested this theory by observing the incidences in the SID without using "negative" POA variable value and the incidences were comparable to the results in the NIS.

Our findings demonstrated that the incidence of delirium doubled every 15 years of age after the age of 60 (Fig. 1, Supplementary Material 1, www.neurores.org). This increase in incidence with age is consistent with other literature [6]. Older age is associated with physiological changes in the brain which puts the patient at an increased susceptibility for developing neurological complications [27]. A similar pattern of increased incidences was also found in people of White race compared to African Americans and Hispanics. However, this observed increase may have been due to bias in reporting, diagnosis, or presentations among different ethnic groups. A detailed multivariable logistic regression of predictors was beyond the scope of this project, and we identify it as the main limitation of this study.

This study was prone to several other limitations. First, hospital claims databases are still prone to errors in coding and reporting. The diagnostic accuracy of delirium ICD-9 codes is still questionable and may not reflect the actual true incidences. Second, we could only track patients during their hospitalization and thus in the majority of cases we could not track to the full 30-day mark used for defining the postoperative period. Third, we also did not assess the effect of undergoing multiple procedures during the same admission on the rates of delirium.

Those findings should aid in improving our understanding of postoperative delirium incidences, and how can incidences vary based on many risk factors, demographics, or definitions. They should also encourage the efforts to improve training for early detection, reporting, and management of this serious neurological complication. Future large-scale studies are still needed to address the clinical and economic impact of postoperative delirium, in addition to focus on how delirium can be prevented, as despite significant advancements in the field of monitoring and diagnostic tools, delirium continue to be commonly encountered complication that is often recognized late. Additionally, utilizing more recent hospital claims databases in the future can be helpful in studying how the numbers change across larger time frames, and whether the change in numbers can be attributed to an actual change in incidences or improved accuracy of reporting.

\section{Conclusions}

This study highlights the size and variation of postoperative incidence across different patient characteristics, risk factors, surgical procedures, and research methods. Delirium occurring in the postsurgical period remains to be a serious potentially preventable problem affecting hospitals worldwide. Implementing additional measures for early detection and prevention can help lessen the morbidity and mortality burden associated with delirium.

\section{Supplementary Material}

Suppl 1. Variation in delirium incidences between SID and NIS across different patient's characteristics.

\section{Acknowledgments}

Many thanks to the University of Pittsburgh IRB office and the Department of Statistics.

\section{Financial Disclosure}

None to declare.

\section{Conflict of Interest}

The authors declare no conflict of interest. The views expressed in this article are the views of the author and do not represent an official position of the University of Alabama at Birmingham or the University of Pittsburgh.

\section{Informed Consent}

Not applicable, because deidentified databases were used.

\section{Author Contributions}

Khaled Al-Robaidi: project leader, hypothesis, data analysis, editing and review; Muruga Loganathan: data analysis and lit- 
erature review; Laith Alhuneafat: manuscript editing and review; Samir S. Al-Ghazawi: manuscript editing; Keith Vogt: review and expertise input; Parthasarathy D. Thirumala: corresponding author, project supervision, review.

\section{Data Availability}

Any inquiries regarding supporting data availability of this study should be directed to the corresponding author.

\section{References}

1. Whitlock EL, Vannucci A, Avidan MS. Postoperative delirium. Minerva Anestesiol. 2011;77(4):448-456.

2. Deiner S, Silverstein JH. Postoperative delirium and cognitive dysfunction. Br J Anaesth. 2009;103(Suppl 1):i4146.

3. Austin CA, O'Gorman T, Stern E, Emmett D, Sturmer T, Carson S, Busby-Whitehead J. Association between postoperative delirium and long-term cognitive function after major nonemergent surgery. JAMA Surg. 2019;154(4):328-334.

4. Liao Y, Flaherty JH, Yue J, Wang Y, Deng C, Chen L. The incidence of delirium after cardiac surgery in the elderly: protocol for a systematic review and meta-analysis. BMJ Open. 2017;7(3):e014726.

5. CDC. National hospital discharge survey: 2010 table, procedures by selected patient characteristics. Centers for Disease Control and Prevention, Atlanta, GA.

6. Inouye SK, Westendorp RG, Saczynski JS. Delirium in elderly people. Lancet. 2014;383(9920):911-922.

7. Rudolph JL, Marcantonio ER. Review articles: postoperative delirium: acute change with long-term implications. Anesth Analg. 2011;112(5):1202-1211.

8. Lat I, McMillian W, Taylor S, Janzen JM, Papadopoulos S, Korth L, Ehtisham A, et al. The impact of delirium on clinical outcomes in mechanically ventilated surgical and trauma patients. Crit Care Med. 2009;37(6):1898-1905.

9. Greene NH, Attix DK, Weldon BC, Smith PJ, McDonagh DL, Monk TG. Measures of executive function and depression identify patients at risk for postoperative delirium. Anesthesiology. 2009;110(4):788-795.

10. Gonzalez M, Martinez G, Calderon J, Villarroel L, Yuri F, Rojas C, Jeria A, et al. Impact of delirium on short-term mortality in elderly inpatients: a prospective cohort study. Psychosomatics. 2009;50(3):234-238.

11. Shehabi Y, Riker RR, Bokesch PM, Wisemandle W, Shintani A, Ely EW, MPH for the SEDCOM (Safety and Efficacy of Dexmedetomidine Compared With Midazolam) Study Group. Delirium duration and mortality in lightly sedated, mechanically ventilated intensive care patients. Crit Care Med. 2010;38(12):2311-2318.

12. Abelha FJ, Luis C, Veiga D, Parente D, Fernandes V, Santos $\mathrm{P}$, Botelho $\mathrm{M}$, et al. Outcome and quality of life in patients with postoperative delirium during an ICU stay following major surgery. Crit Care. 2013;17(5):R257.

13. Ansaloni L, Catena F, Chattat R, Fortuna D, Franceschi C, Mascitti P, Melotti RM. Risk factors and incidence of postoperative delirium in elderly patients after elective and emergency surgery. Br J Surg. 2010;97(2):273-280.

14. Rockwood K, Cosway S, Carver D, Jarrett P, Stadnyk K, Fisk J. The risk of dementia and death after delirium. Age Ageing. 1999;28(6):551-556.

15. Martin CM, Hill AD, Burns K, Chen LM. Characteristics and outcomes for critically ill patients with prolonged intensive care unit stays. Crit Care Med. 2005;33(9):19221927; quiz 1936.

16. NIH - HCUP. Published 2010. https://www.hcup-us.ahrq. gov/nisoverview.jsp.

17. Kazmierski J, Kowman M, Banach M, Fendler W, Okonski P, Banys A, Jaszewski R, et al. Incidence and predictors of delirium after cardiac surgery: results from the IPDACS study. J Psychosom Res. 2010;69(2):179-185.

18. Brown CHt, LaFlam A, Max L, Wyrobek J, Neufeld KJ, Kebaish KM, Cohen DB, et al. Delirium after spine surgery in older adults: incidence, risk factors, and outcomes. J Am Geriatr Soc. 2016;64(10):2101-2108.

19. Shi CM, Wang DX, Chen KS, Gu XE. Incidence and risk factors of delirium in critically ill patients after non-cardiac surgery. Chin Med J (Engl). 2010;123(8):993-999.

20. Norkiene I, Ringaitiene D, Misiuriene I, Samalavicius R, Bubulis R, Baublys A, Uzdavinys G. Incidence and precipitating factors of delirium after coronary artery bypass grafting. Scand Cardiovasc J. 2007;41(3):180-185.

21. Yang Q, Wang J, Huang X, Xu Y, Zhang Y. Incidence and risk factors associated with postoperative delirium following primary elective total hip arthroplasty: a retrospective nationwide inpatient sample database study. BMC Psychiatry. 2020;20(1):343.

22. Kales HC, Kamholz BA, Visnic SG, Blow FC. Recorded delirium in a national sample of elderly inpatients: potential implications for recognition. J Geriatr Psychiatry Neurol. 2003;16(1):32-38.

23. Dworsky JQ, Shellito AD, Childers CP, Copeland TP, Maggard-Gibbons M, Tan HJ, Saliba D, et al. Association of geriatric events with perioperative outcomes after elective inpatient surgery. J Surg Res. 2021;259:192-199.

24. Soundhar A, Udesh R, Mehta A, Schindler J, Jeevanantham V, Gleason T, Thirumala PD. Delirium following transcatheter aortic valve replacement: national inpatient sample analysis. J Cardiothorac Vasc Anesth. 2017;31(6): 1977-1984.

25. Armstrong SC, Cozza KL, Watanabe KS. The misdiagnosis of delirium. Psychosomatics. 1997;38(5):433-439.

26. McCoy TH, Jr., Snapper L, Stern TA, Perlis RH. Underreporting of delirium in statewide claims data: implications for clinical care and predictive modeling. Psychosomatics. 2016;57(5):480-488.

27. Esiri MM.Ageing and the brain.J Pathol.2007;211(2):181187. 